\title{
A randomized, controlled trial comparing the clinical outcomes of 3D versus 2D laparoscopic hysterectomy
}

\author{
Taejong Song ${ }^{1}$, Du-young Kang ${ }^{2}$ \\ ${ }^{1}$ Department of Obstetrics and Gynecology, Kangbuk Samsung Hospital, Sungkyunkwan University, School of Medicine, Seoul, \\ Republic of Korea \\ 2Department of Thoracic and Cardiovascular Surgery, Kangbuk Samsung Hospital, Sungkyunkwan University, School of Medicine, \\ Seoul, Republic of Korea
}

Videosurgery Miniinv 2022; 17 (1): 127-133 DOI: https://doi.org/10.5114/wiitm.2021.105724

\begin{abstract}
Introduction: There have been a few clinical studies on the use of three-dimensional (3D) laparoscopy with different results.

Aim: To compare the surgical outcomes of 3D versus two-dimensional (2D) laparoscopic hysterectomy for benign or premalignant gynecologic diseases.

Material and methods: In this double-blind trial, 68 patients were randomly assigned to either the 3D or 2D groups at a 1: 1 ratio. The only difference between the two groups was the laparoscopic vision system used. The primary outcome was operative blood loss and operative time. The other surgical outcomes including failure of the intended surgery, length of hospital stay, and operative complications were also assessed.

Results: The baseline characteristics did not statistically significantly differ between the groups. The mean operative blood loss was not significantly different between the 3D group $(74.4 \pm 51.6 \mathrm{ml})$ and the 2D group $(79.2 \pm 55.4 \mathrm{ml})$ $(p=0.743)$. The operative time was similar in both groups $(84.5 \pm 20.5 \mathrm{~min}$ vs. $87.8 \pm 24.4 \mathrm{~min}, p=0.452)$. Moreover, no differences were observed between the groups in other surgical outcomes.

Conclusions: The 3D imaging system had no surgical advantage in laparoscopic hysterectomy for benign or premalignant gynecologic diseases. However, 3D laparoscopy did not have any negative effects on surgical outcomes and did not increase the surgical risk.
\end{abstract}

Key words: laparoscopy, hysterectomy, three-dimensional, two-dimensional.

\section{Introduction}

Laparoscopic hysterectomy is currently considered the gold standard for the treatment of benign uterine or premalignant diseases [1]. This surgery was performed for the first time by Harry Reich in 1988 [2] and in the past years, many studies have shown the advantages of the laparoscopic approach, with decreases in postoperative complication rates, less operative bleeding, less postoperative pain, and shorter postoperative hospital stays compared to abdominal hysterectomy [3].

However, laparoscopic surgery is more difficult to learn and requires different psychomotor skills than open surgery. In fact, the surgeons have to work in a three-dimensional (3D) space but are guided by two-dimensional (2D) images $[4,5]$. Due to the lack of depth perception and spatial orientation in the traditional 2D imaging system, 3D laparoscopy was developed as an alternative to conventional 2D

\section{Address for correspondence}

Taejong Song MD, PhD, Department of Obstetrics and Gynecology, Kangbuk Samsung Hospital, Sungkyunkwan University,

School of Medicine, 29 Saemunan-ro, Jongno-gu, Seoul 03181, Republic of Korea, phone: +82-2-2001-2058, fax: +82-2-2001-1102,

e-mail: taejong.song@gmail.com 
laparoscopy [6]. Although 3D technology was introduced in the early 1990s, the equipment is still not standardly available in hospitals because of initial reports of side effects when using 3D imaging systems, poor image resolution, and higher cost [6]. There are a few clinical studies on the use of 3D with different results $[7,8]$.

\section{Aim}

Therefore, the present study aimed to compare the surgical outcomes of 3D versus 2D laparoscopic hysterectomy.

\section{Material and methods}

\section{Patients}

A randomized, controlled trial that evaluated the effect of a 3D scope in laparoscopic hysterectomy was prospectively conducted between September 2019 and September 2020 at the authors' institution. The protocol was approved by the Institutional Review Board and registered with ClinicalTrials. gov (identifier: NCT04070872; date of trial registration: August 23, 2019, https://clinicaltrials.gov/ ct2/show/NCT04070872). Women with indications for laparoscopic hysterectomy for benign or premalignant gynecologic conditions were invited to participate in the study. The inclusion criteria were as follows: age between 18 and 80 years, American Society of Anesthesiologists Physical Status classification I-II, and the absence of pregnancy or lactation at the time of surgery. The exclusion criterion was any suspicious findings of malignant gynecologic disease.

The patients were randomly assigned to the $3 \mathrm{D}$ group and the 2D group at a $1: 1$ ratio using a random permuted-block randomization algorithm via an interactive web-based response system (http:// www.randomization.com). A study coordinator who was unaware of the personal and medical information of the patients in an office distant from the hospital prepared sequentially numbered, opaque, sealed envelopes containing the assigned intervention to ensure that the sequence was concealed before the study began. The study investigators called the study coordinator on the day of surgery for randomization. The study was performed in accordance with the protocol, and all patients provided written informed consent before participation.

\section{Laparoscopic devices}

All surgical procedures were performed by one surgeon, who had performed more than 1500 laparoscopic hysterectomy procedures to control the variability in surgical skill. The laparoscopic port (or trocar) placement was determined according to the patient's condition and needs. For the laparoscopic camera system, a 10-mm ENDOEYE FLEX 3D Deflectable Videoscope LTF-190-10-3D (Olympus Corp., Hamburg, Germany) and a 10-mm 30 IDEAL EYES Laparoscope (Stryker, Kalamazoo, MI, USA) camera were used in the $3 \mathrm{D}$ group and the $2 \mathrm{D}$ group, respectively. The laparoscopic equipment and the operative procedural steps were the same in every patient to ensure a standardized approach. The only difference between the two groups was the laparoscopic camera used.

\section{Surgical techniques}

The operative technique used for laparoscopic hysterectomy was previously described in detail [9]. In brief, general anesthesia with endotracheal intubation was achieved and the patients were placed in the deep Trendelenburg position. After uterine sounding and cervical dilation, a RUMI uterine manipulator with a Koh Colpotomizer and Colpo-Pneumo Occluder (Cooper Surgical, Inc., Trumbull, CT, USA) was fixed onto the cervix to effectively construct a surgical field. Using an open Hasson approach, a $1.5-2-\mathrm{cm}$ vertical incision was made within the umbilicus. Next, after pneumoperitoneum was created following insufflation with carbon dioxide to a pressure of $11 \mathrm{~mm} \mathrm{Hg}$, a laparoscope was inserted through the umbilical port. While the uterine body was retracted medially using either laparoscopic forceps or a myoma screw, the adnexal pedicle, round ligament, and broad ligament were transected with LigaSure (Valleylab, Boulder, CO, USA). Thereafter, the vesicouterine peritoneal fold was identified, and the bladder was mobilized by blunt and sharp dissection using LigaSure until the anterior vagina was identified. The uterine vessels were skeletonized, sealed, and transected using LigaSure. The cardinal and uterosacral ligaments were then transected. This procedure was then repeated on the opposite side. A circumferential colpotomy was performed with a monopolar electrical device over the Colpotomizer cup. The specimen was removed via the vagina, and uterine morcellation was performed with a knife, if 
necessary. Vaginal cuff closure was achieved laparoscopically. After carefully examining the bleeding and washing the pelvic cavity, the procedure was completed. The peritoneum and fascia were then approximated and closed using 1-0 Vicryl sutures (Ethicon, Somerville, NJ, USA). A liquid topical skin adhesive (Dermabond, Ethicon) was applied to close the incision. The patients were discharged from the hospital after the restoration of bowel activity, in the absence of postoperative fever, when they no longer needed narcotic analgesics, and could successfully ambulate. All patients were scheduled for check-up examinations at 1 week and 1 month after surgery.

\section{Outcome measures}

The primary outcome was operative blood loss and operative time. Operative blood loss was measured by the anesthesiologists after defining it as the difference between the total amount of suction and irrigation plus the difference between the total gauze weight before and after surgery. Operative time was defined as the time from incision to closure of the skin. Surgery was considered to have failed if the surgeon was required to use one or more additional ports or convert to laparotomy. The length of hospitalization (defined as the number of days from the operation day to the day of discharge), intraoperative complications (defined as major vessel injury, bowel injury, urinary tract injury, or any other severe unplanned events), and postoperative complications (defined as grade III or higher complications occurring within 30 days of surgery according to the Clavien-Dindo classification [10]) were assessed.

\section{Statistical analysis}

The present study was designed as a non-equality test, the hypothesis of which was to establish that 3D laparoscopic hysterectomy is not equal to conventional 2D laparoscopy in terms of surgical outcome. Therefore, the sample size was calculated according to the difference in operative blood loss, which was collected retrospectively from 30 consecutive patients who underwent conventional 2D laparoscopic hysterectomy before this study, showing an operative blood loss of $83.5 \pm 57.8 \mathrm{ml}$ (authors' unpublished data). We estimated that 34 patients would be required per group to yield a type I error of 0.05 , a power of $80 \%$, and a predicted dropout rate of $10 \%$ to detect a difference of $41.8 \mathrm{ml}(50 \%$ difference in the operative blood loss), which was considered clinically relevant, between the groups. No interim analysis was planned or performed.

SPSS software 23.0 was used for the statistical analyses. All analyses were performed according to the intention-to-treat principle. For continuous variables, the data are presented as the mean \pm standard deviation (SD) or median (interquartile range (IQR)) after verifying the normal distribution of the data. For categorical variables, data are presented as frequency (percent). The baseline characteristics, primary, and secondary outcomes were compared between the two groups using Student's $t$-test or the Mann-Whitney $U$ test for continuous variables, and the $\chi^{2}$ test or Fisher's exact test for categorical variables, as appropriate. A $p$-value of $<0.05$ was considered statistically significant.

\section{Results}

Enrollment took place between September 2019 and September 2019. Follow-up visits concluded in December 2020. Of the 77 candidates who were asked to participate in this trial, 9 were excluded because of a suspicious premalignant ovarian tumor $(n=1)$, planned concomitant surgery (i.e., urinary incontinence surgery or cholecystectomy) $(n=2)$, a change in treatment from hysterectomy to myomectomy according to the patient's request $(n=3)$, and refusal to participate $(n=3)$. Therefore, 68 patients were randomly assigned to the $3 \mathrm{D}$ group or the $2 \mathrm{D}$ group (Figure 1 ). After randomization or surgery, none of the patients changed their assigned groups or stopped participating in the trial.

The baseline characteristics of both groups are shown in Table I. The mean age and body mass index of the patients were $45.5 \pm 5.4$ years and $23.7 \pm 3.6$ $\mathrm{kg} / \mathrm{m}^{2}$, respectively, with no significant differences between the groups. The other baseline characteristics of a history of abdominal surgery, parity, uterine size, preoperative hemoglobin level, laparoscopic approach, mode of hysterectomy, indication for hysterectomy, and procedure performed were also not different between the groups (all, $p>0.005$ ).

Table II shows the primary and other surgical outcomes. The mean operative blood loss was not statistically different between the 3D group $(74.4 \pm 51.6 \mathrm{ml})$ and the $2 \mathrm{D}$ group $(79.2 \pm 55.4 \mathrm{ml})(p=0.743)$. The operative time was similar in both groups (84.5 $\pm 20.5 \mathrm{~min}$ vs. $87.8 \pm 24.4 \mathrm{~min}, p=0.452$ ). Moreover, 


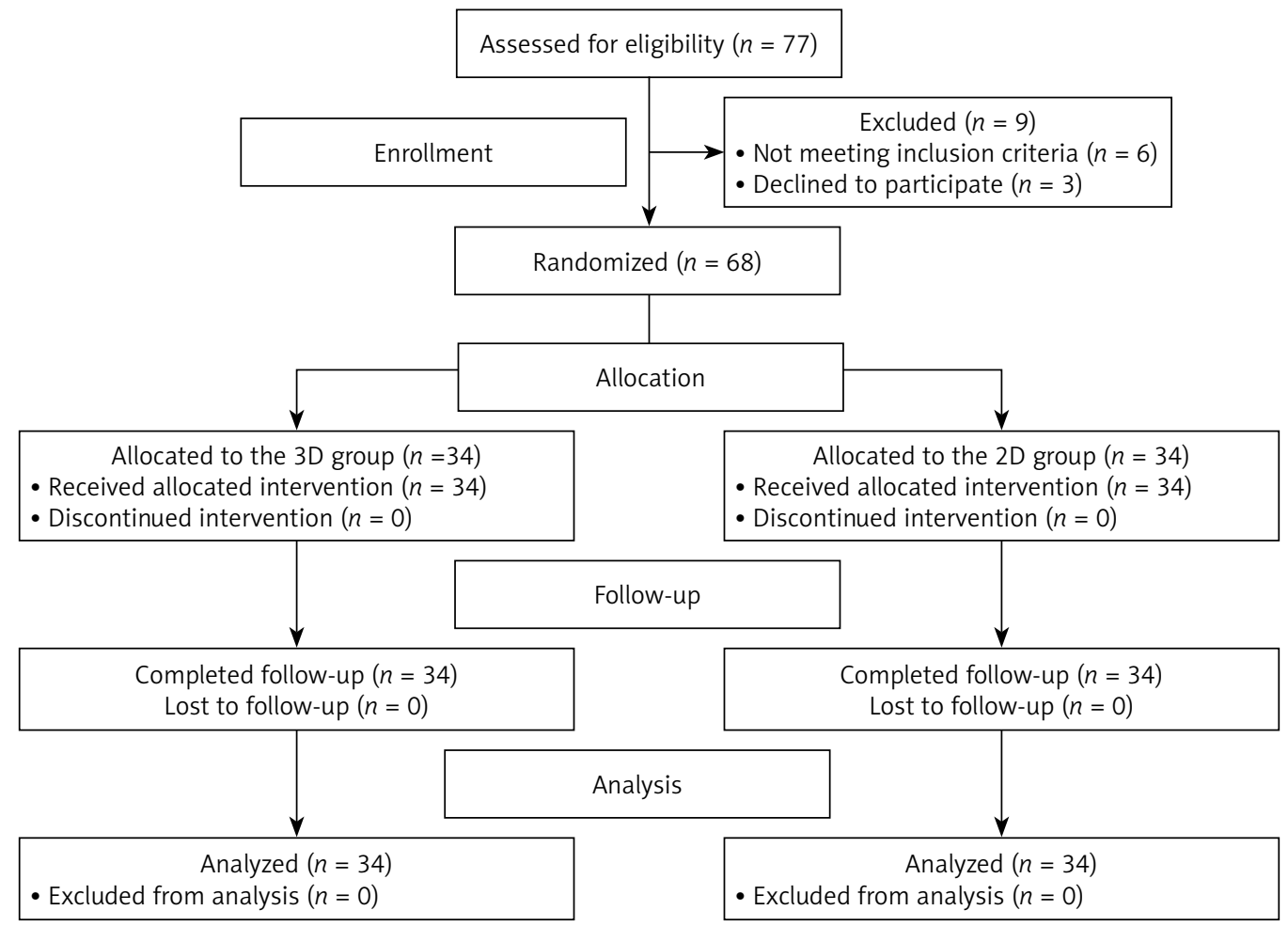

Figure 1. CONSORT diagram of the study

no differences were observed between the groups in other surgical outcomes including changes in serum hemoglobin (defined as the difference between preoperative hemoglobin levels and hemoglobin levels at the first postoperative day), transfusion, the weight of the extracted uterus, adhesiolysis at the time of surgery, failure of the intended surgery, postoperative pain score (measured on a visual analog scale (0-10 scale), ranging from "no pain" to "pain as bad as it could be"), length of hospitalization, intraoperative complications, and postoperative complications. One patient in the 2D group experienced a postoperative wound complication requiring resuturing 2 weeks after surgery.

\section{Discussion}

We conducted this randomized controlled trial to test the hypothesis that a 3D imaging system could show favorable surgical outcomes in laparoscopic hysterectomy. The main finding of this trial was that 3D laparoscopy did not affect operative blood loss or operative time in patients who underwent laparoscopic hysterectomy for benign gynecologic diseases. We also found that other surgical outcomes were not influenced by the laparoscopic vision system (3D vs. 2D). We assert that this study is valuable to laparoscopists who are interested in 3D laparoscopy or minimally invasive surgery.

This study demonstrated that 3D laparoscopy did not improve the surgical outcomes of laparoscopic hysterectomy for benign or premalignant gynecologic diseases. In the field of gynecologic surgery, three previous studies have compared $3 \mathrm{D}$ versus $2 \mathrm{D}$ laparoscopy [8, 11, 12]. Yazawa et al. reported the surgical outcomes of $3 \mathrm{D}$ versus $2 \mathrm{D}$ laparoscopic hysterectomy for benign gynecologic diseases [8]. They retrospectively compared 47 earlier laparoscopic hysterectomies using 2D laparoscopy (performed between July 2013 and October 2014) with 47 later laparoscopic hysterectomies using 3D laparoscopy (performed between November 2014 and December 2015). The 3D group had operative blood loss statistically similar to the 2D group (192 \pm 174 vs. $161 \pm 147 \mathrm{ml}, p=0.345$ ) [8]. No differences in other perioperative outcomes or postoperative complications were observed between the two groups. The surgeons did not report any symptoms attributable to the 3D imaging system such as dizziness, eyestrain, nausea, or headache. Fanfani et al. performed 
Table I. Baseline characteristics

\begin{tabular}{|c|c|c|c|}
\hline Parameter & $3 \mathrm{D}$ group $(n=34)$ & $2 \mathrm{D}$ group $(n=34)$ & $P$-value \\
\hline Age [years] & $45.4 \pm 5.1$ & $45.6 \pm 5.8$ & 0.602 \\
\hline Body mass index [kg/m²] & $23.6 \pm 3.7$ & $23.8 \pm 3.6$ & 0.897 \\
\hline History of abdominal surgery & $13(38.2 \%)$ & $12(35.3 \%)$ & 0.801 \\
\hline Parity: & & & 0.720 \\
\hline Nulliparous & $4(11.8 \%)$ & $5(14.7 \%)$ & \\
\hline Parous & $30(88.2 \%)$ & $29(85.3 \%)$ & \\
\hline \multicolumn{4}{|l|}{ Uterine axis [cm]: } \\
\hline Long & $11.0 \pm 1.8$ & $10.9 \pm 1.9$ & 0.631 \\
\hline Short & $8.1 \pm 2.0$ & $8.0 \pm 1.8$ & 0.482 \\
\hline Preoperative hemoglobin [mg/dl] & $10.2 \pm 1.8$ & $10.3 \pm 1.6$ & 0.739 \\
\hline Laparoscopic approach, initial intent: & & & $>0.999$ \\
\hline Single-port laparoscopy & $33(97.1 \%)$ & $33(97.1 \%)$ & \\
\hline Multi-port laparoscopy & $1(2.9 \%)$ & $1(2.9 \%)$ & \\
\hline Mode of hysterectomya: & & & $>0.999$ \\
\hline LAVH & $0(0 \%)$ & $1(2.9 \%)$ & \\
\hline$\overline{\mathrm{TLH}}$ & $34(100 \%)$ & $33(97.1 \%)$ & \\
\hline Indication for hysterectomy (\%): & & & 0.837 \\
\hline Uterine myoma or adenomyosis & $30(88.2 \%)$ & $29(85.3 \%)$ & \\
\hline Cervical intraepithelial neoplasia & $3(8.8 \%)$ & $3(8.8 \%)$ & \\
\hline Endometrial pathology & $1(2.9 \%)$ & $2(5.9 \%)$ & \\
\hline Procedure performed: & & & 0.525 \\
\hline Hysterectomy alone & $27(79.4 \%)$ & $29(85.3 \%)$ & \\
\hline With adnexal surgery ${ }^{b}$ & $7(20.6 \%)$ & $5(14.7 \%)$ & \\
\hline
\end{tabular}

The values are presented as frequency (percent), mean \pm standard deviation (SD) or median (interquartile range (IQR) after verifying the normal distribution of the data. 'a Laparoscopic hysterectomy was subclassified according to the method of securing and dividing the uterine artery: laparoscopically assisted vaginal hysterectomy (LAVH) and total laparoscopic hysterectomy $(T L H)$ refer to when the uterine vessels were secured vaginally and laparoscopically, respectively. ${ }^{b} A d n e x a l$ procedures did not include opportunistic salpingectomy. Opportunistic salpingectomy was included in the "Hysterectomy alone" category. Opportunistic salpingectomy is the removal of the fallopian tubes for the primary prevention of ovarian cancer in a woman already undergoing pelvic surgery for another indication.

Table II. Primary and other clinical outcomes

\begin{tabular}{|lccc|}
\hline Parameter & 3D group $(n=34)$ & 2D group $(n=34)$ & $P$-value \\
\hline Operative blood loss [ml] & $74.4 \pm 51.6$ & $79.2 \pm 55.4$ & 0.743 \\
\hline Operative time [min] & $84.5 \pm 20.5$ & $87.8 \pm 24.4$ & 0.452 \\
\hline Change in serum hemoglobin [g/dl] & $1.4 \pm 0.8$ & $1.3 \pm 1.1$ & 0.588 \\
\hline Transfusion & $1(\%)$ & $1(\%)$ & $>.999$ \\
\hline Weight of extracted uterus [g] & $350.3 \pm 155.6$ & $342 \pm 163.4$ & 0.552 \\
\hline Adhesiolysis at the time of surgery & $10(29.4 \%)$ & $8(23.5 \%)$ & 0.582 \\
\hline Failure of intended surgery: & $1(2.9 \%)$ & $2(5.9 \%)$ & $>0.999$ \\
\hline Additional port insertion & 1 & 1 & \\
\hline Conversion to LAVH from TLH & 0 & 0 & 0.552 \\
\hline Conversion to open surgery & 0 & $3.4 \pm 1.4$ & 0.392 \\
\hline Postoperative pain scorea: & $3.3 \pm 1.2$ & $2.7 \pm 1.1$ & 0.678 \\
\hline At 12 h after surgery & $2.6 \pm 0.8$ & $2.0 \pm 0.2$ & $>0.999$ \\
\hline At 24 h after surgery & $2.0 \pm 0.3$ & & 0 \\
\hline Length of hospitalization [days] & 0 & $1(\%)$ & \\
\hline Operative complications: & 0 & & \\
\hline Intraoperative complications & & & \\
\hline Postoperative complications & & & \\
\hline
\end{tabular}

aPostoperative pain score was measured using a visual analog scale (0-10 points), ranging from "no pain" to "pain as bad as it could be". 
a randomized controlled trial comparing the outcomes of 3D versus 2D laparoscopic hysterectomy with lymphadenectomy in 90 patients with endometrial or cervical cancer [11]. The surgical outcomes such as operative time (110 min, range: 25-393 vs. $108 \mathrm{~min}$, range: $30-345, p=0.593)$, operative blood loss (126 ml, range: 0-500 vs. $142 \mathrm{~min}$, range: 0-650, $p=0.982$ ), and postoperative complications were similar between both the $2 \mathrm{D}$ and $3 \mathrm{D}$ groups [11]. Lui et al. conducted a randomized controlled trial in 75 patients undergoing laparoscopic ovarian cystectomy and evaluated whether 3D laparoscopy had any advantage over 2D laparoscopy [12]. There were no significant differences between the $2 \mathrm{D}$ and $3 \mathrm{D}$ groups regarding operative time (47.6 \pm 32.0 vs. $51.6 \pm 36.2 \mathrm{~min}, p=0.198)$ and operative blood loss $(55.1 \pm 64.8$ vs. $58.2 \pm 56.2 \mathrm{ml}, p=0.825)$. The participating surgeons in the 2D group reported nausea, dizziness, ocular fatigue, and blurring of vision less frequently than did those in the $3 \mathrm{D}$ group $(5.3 \%$ vs. 45.9\%; $p<0.001$ ) [12]. Taken together, we believe that 3D vision in the field of laparoscopic hysterectomy does not have a positive impact on the surgeon's performance.

In the present study, no differences were observed between the $3 \mathrm{D}$ and $2 \mathrm{D}$ groups in operative blood loss and operative time. These findings can be attributed to the following three reasons. First, laparoscopic hysterectomy is generally a complex procedure, but it is not accompanied by very difficult techniques (i.e., retroperitoneal space dissection, reanastomosis, or intensive suturing). Because 3D laparoscopy mainly improves the depth of perception, leading to better visibility $[7,13]$, the 3D vision system may be valuable for more complex gynecologic procedures including myomectomy or gynecologic cancer surgery. Second, conventional 2D laparoscopy did not cause side effects related to the 3D images. 3D vision systems could cause side effects for surgeons, such as eye strain, headaches, dizziness, and visual discomfort [14-16]. Third, all operations were performed by one fully experienced surgeon who had performed more than 1500 laparoscopic hysterectomy procedures prior to this study. Previous studies have shown that 3D laparoscopy was beneficial for less experienced surgeons $[11,17,18]$.

There were several limitations to this study. First, the main limitation of this study is that the procedures were performed by a single experienced surgeon. Thus, our results may not be applicable to other surgeons. Second, we did not assess the side effects of stereoacuity in this study. Han et al. reported that $67 \%$ of surgeons experienced visually induced motion sickness (VIMS) during their first 3D laparoscopy case [19]. However, the incidence and severity of VIMS dramatically decreased from the second case onward. Finally, this study was not blinded from the surgeon (study investigators) because this would have been impossible given the nature of the study. However, patients and outcome assessors were unaware of the allocation information.

\section{Conclusions}

The 3D imaging system had no surgical advantage in laparoscopic hysterectomy for benign gynecologic diseases. However, 3D laparoscopy did not have any negative effects on surgical outcomes and did not increase the surgical risk. Considering that more complex procedures such as suturing and adhesiolysis might be easier to perform with 3D laparoscopy than with 2D laparoscopy, additional studies in various laparoscopic settings (more difficult hysterectomies, severe adhesion cancer surgery, and multiple surgeons with different surgical skills) are warranted to validate the current results.

\section{Acknowledgments}

Taejong Song and Du-young Kang contributed equally to this study.

Institutional review board number 2019-07-042, September 2019, ClinicalTrials.gov ID NCT04070872, https://clinicaltrials.gov/ct2/show/NCT04070872.

\section{Conflict of interest}

The authors declare no conflict of interest.

\section{References}

1. Margulies SL, Vargas MV, Denny K et al. Comparing benign laparoscopic and abdominal hysterectomy outcomes by time. Surg Endosc 2020; 34: 758-69.

2. Reich H, DeCaprio J, McGlynn F. Laparoscopic hysterectomy. J Gynecol Surg 1989; 5: 213-6.

3. Louie M, Strassle PD, Moulder JK, et al. Uterine weight and complications after abdominal, laparoscopic, and vaginal hysterectomy. Am J Obstet Gynecol 2018; 219: 480e481-8.

4. Tanagho YS, Andriole GL, Paradis AG, et al. $2 \mathrm{D}$ versus $3 \mathrm{D}$ visualization: impact on laparoscopic proficiency using the fundamentals of laparoscopic surgery skill set. J Laparoendosc Adv Surg Tech A 2012; 22: 865-70. 
5. Storz P, Buess GF, Kunert W, Kirschniak A. 3D HD versus 2D HD: surgical task efficiency in standardised phantom tasks. Surg Endosc 2012; 26: 1454-60.

6. Kunert W, Storz P, Kirschniak A. For 3D laparoscopy: a step toward advanced surgical navigation: how to get maximum benefit from 3D vision. Surg Endosc 2013; 27: 696-9.

7. Sorensen SM, Savran MM, Konge L, Bjerrum F. Three-dimensional versus two-dimensional vision in laparoscopy: a systematic review. Surg Endosc 2016; 30: 11-23.

8. Yazawa H, Takiguchi K, Imaizumi K, et al. Surgical outcomes of total laparoscopic hysterectomy with 2-dimensional versus 3-dimensional laparoscopic surgical systems. Fukushima J Med Sci 2018; 64: 38-45.

9. Song T, Kim MK, Kim ML, et al. A randomized comparison of laparoendoscopic single-site hysterectomies: total laparoscopic hysterectomy versus laparoscopically assisted vaginal hysterectomy. J Laparoendosc Adv Surg Tech A 2015; 25: 541-7.

10. Clavien PA, Barkun J, de Oliveira ML, et al. The Clavien-Dindo classification of surgical complications: five-year experience. Ann Surg 2009; 250: 187-96.

11. Fanfani F, Rossitto C, Restaino S, et al. How technology can impact surgeon performance: a randomized trial comparing 3-dimensional versus 2-dimensional laparoscopy in gynecology oncology. J Minim Invasive Gynecol 2016; 23: 810-7.

12. Lui MW, Cheung VY. Three-dimensional versus two-dimensional laparoscopy for ovarian cystectomy: a prospective randomised study. Hong Kong Med J 2018; 24: 245-51.

13. Liang H, Liang W, Lei Z, et al. Three-dimensional versus two-dimensional video-assisted endoscopic surgery: a meta-analysis of clinical data. World J Surg 2018; 42: 3658-68.

14. Chan AC, Chung SC, Yim AP, et al. Comparison of two-dimensional vs three-dimensional camera systems in laparoscopic surgery. Surg Endosc 1997; 11: 438-40.

15. Mueller MD, Camartin C, Dreher E, Hanggi W. Three-dimensional laparoscopy. Gadget or progress? A randomized trial on the efficacy of three-dimensional laparoscopy. Surg Endosc 1999; 13: 469-72.

16. Hanna GB, Shimi SM, Cuschieri A. Randomised study of influence of two-dimensional versus three-dimensional imaging on performance of laparoscopic cholecystectomy. Lancet 1998; 351: 248-51.

17. Alaraimi B, El Bakbak W, Sarker S, et al. A randomized prospective study comparing acquisition of laparoscopic skills in three-dimensional (3D) vs. two-dimensional (2D) laparoscopy. World J Surg 2014; 38: 2746-52.

18. Cicione A, Autorino R, Breda A, et al. Three-dimensional vs standard laparoscopy: comparative assessment using a validated program for laparoscopic urologic skills. Urology 2013; 82: $1444-50$.

19. Han YG, Song T, Kang H, et al. Incidence and severity of Visually Induced motion Sickness during 3D laparoscopy In Operators who had No experience with it (VISION). Videosurgery Miniinv 2020; 15: 283-9.

Received: 15.03.2021, accepted: 30.03.2021. 